\title{
Estabilización escafolunar mediante la técnica «3LT». Indicaciones, técnica quirúrgica y resultados
}

\author{
M. Garcia-Elias ${ }^{(1)}$, A. Lluch ${ }^{(1)}$, J. K. Stanley ${ }^{(2)}$ \\ ${ }^{(1)}$ INSTITUT KAPLAN. \\ Cirugía de la Mano y la Extremidad Superior. Barcelona (España). \\ ${ }^{(2)}$ DePARTMENT OF HAND AND UPPER LIMB SURGERY. \\ Wrightington Hospital For JoINT Disease. WigAn, ENGLAND (REINo UNIDo).
}

Correspondencia

Dr. Marc Garcia-Elias

Institut Kaplan

Paseo de la Bonanova, 9, 2을

08022 Barcelona (España)

E-mail: garciaelias@infonegocio.com

La rotura traumática o degenerativa de los ligamentos escafolunares suele inducir un cuadro de inestabilidad progresiva del carpo en el curso de la cual el escafoides sufre un colapso en flexión y pronación, mientras que el semilunar y el piramidal adoptan una postura anómala en extensión, supinación y traslación cubital. Si la lesión es diagnosticada en la fase aguda, y sus características permiten una reparación eficaz, los resultados que pueden obtenerse suelen ser satisfactorios. Lamentablemente, las roturas escafolunares suelen pasar desapercibidas de entrada con lo que aparece la denominada disfunción escafolunar crónica caracterizada por pérdida de fuerza, disminución progresiva de la movilidad, y la presencia de resaltes esporádicos dolorosos. Con el tiempo dicha disfunción genera cambios artrósicos siguiendo un patrón característico, denominado SLAC («scaphoid-lunate advanced collapse»). El tratamiento de la disfunción escafolunar crónica depende del grado evolutivo en que se encuentre. En este artículo se describe un algoritmo que resulta útil en el momento de diseñar un tratamiento para cada caso concreto de inestabilidad escafolunar. Uno de los grados de inestabilidad escafolunar más difíciles de tratar es aquel en el cual el ligamento escafolunar dorsal es irreparable, y sin embargo la pérdida de alineación es reducible y no existe todavía artrosis. Para este tipo de inestabilidad (grado 4, según el algoritmo que proponemos), la muñeca pue-
Traumatic or degenerative ruptures of the scapholunate ligaments may induce progressive carpal instability, with the scaphoid collapsing into flexion and pronation, while the lunate and triquetrum undergo abnormal extension, supination and ulnar translation. Acceptable results may be obtained if the healing potential of the injury is good and if the repair is done early. Unfortunately, this type of ligament lesions are often missed at presentation for a carpal dysfunction characterized by painful snapping and weak grip strength appears. Down the line, such dysfunction tends to deteriorate the periscaphoid cartilage inducing osteoarthritis following a characteristic pattern, the so-called SLAC wrist («scaphoid-lunate advanced collapse»). Obviously, treatment of scapholunate instability is stage-dependent. In this article we propose a staging system that helps deciding how manage each individual case.

From a treatment viewpoint, one of the most problematic stages of the injury is when the ligament repair is not feasible, and yet the subluxation is easily reducible, and no cartilage defects are present. For those cases (stage 4 according to the proposed staging system) the so-called 3LT tenodesis procedure («Three-ligament-tenodesis») is recommended. This paper reviews the surgical technique, as well as the results obtained in 37 patients, with an average 47 months follow-up, who exhibited more than 
de recuperar un buen grado de función indolora mediante la técnica 3LT («Three-ligament-tenodesis»). En este artículo se detalla este tipo de tenodesis, así como los resultados obtenidos en 38 pacientes, con un seguimiento promedio de 47 meses, destacando una movilidad global superior al $70 \%$ de la contralateral, una fuerza de prensión del $65 \%$, y mínimas molestias residuales en la gran mayoría de casos.

Palabras clave: carpo, escafoides, disociación escafolunar, tenodesis
$70 \%$ of the contralateral range of motion, $65 \%$ grip strength, and minimal residual discomfort in the vast majority.

Key words: carpus, scaphoid, scapholunate dissociation, tenodesis

Rev. Iberam. Cir. Mano - Vol. 33 - Núm. 67 - Noviembre 2005 (9-21)

\section{INTRODUCCIÓN}

A pesar de su apariencia banal en su fase temprana, si una disociación escafolunar (E-L) de origen traumático o degenerativo no es correctamente diagnosticada $\mathrm{y}$ tratada puede tener consecuencias funcionales importantes a medio y largo plazo. Desgraciadamente, el porcentaje de lesiones que pasan desapercibidas en la práctica diaria sigue siendo inaceptablemente alto $^{1-3}$. Con el tiempo, los ligamentos distendidos o rotos pierden su potencial cicatrizante al sufrir un proceso de retracción y/o necrosis de su extremo libre. Ello facilita la aparición de fenómenos de distensión progresiva de una serie de estructuras estabilizadoras como puedan ser las cápsulas dorsal radioescafoidea y palmar escafo-trapecio-trapezoidea, así como los ligamentos escafoides-trapecio-trapezoide, escafoides-hueso grande y radio-escafoides-hueso grande. Ello conlleva un aumento de la subluxación rotatoria del escafoides en flexión-pronación, llevando a su polo proximal a articularse con el borde dorsoradial de la apófisis estiloides radial ${ }^{4}$. A medida que el escafoides pierde su normal alineación carpiana, las articulaciones vecinas desarrollan patrones de movilidad anómalos con lo que aparece una marcada inestabilidad caracterizada por la presencia de dolor más o menos intenso y una pérdida progresiva de movilidad y de fuerza. La muñeca suele ceder ante cargas axiales en forma de resaltes dolorosos característicos ${ }^{5-8}$. Con el tiempo, la disfunción resultante genera cambios artrósicos siguiendo un patrón típico, denominado SLAC («scaphoid-lunate advanced collapse») $)^{3}$.

Múltiples soluciones quirúrgicas han sido propuestas para el tratamiento de la inestabilidad E-L: capsulo-tenodesis ${ }^{9-20}$, transferencias hueso-ligamento-hueso ${ }^{21,22}$, artrodesis parcia$\operatorname{les}^{23-25}$, o carpectomia proximal ${ }^{26}$. El objetivo de todas ellas es lograr una función aceptable con la morbilidad más baja posible en términos de reducción de la movilidad y en capacidad de sostener cargas fisiológicas sin que la muñeca ceda en forma de resalte doloroso. Si bien es obvio que ninguna de esas técnicas está indicada para todos los grados de inestabilidad, no parece existir un consenso en cuanto a cual es mejor para cada tipo de lesión. Parece razonable que el tratamiento no sólo debe tener en cuenta las demandas individuales de cada paciente, sino que además debe ser proporcional al grado de desestabilización existente en función de las características propias de cada lesión y de la cronicidad del cuadro a tratar. Para ello, proponemos un método de análisis de las inestabilidades E-L que nos ha sido útil en el momento de tomar decisiones terapéuticas en este tipo de lesiones. Así mismo, presentamos la técnica quirúrgica 3LT («three-ligament tenodesis») que hemos diseñado y aplicado satisfactoriamente en las inestabilidades reducibles, no reparables y sin cambios artrósicos de las articulaciones carpianas. 


\section{ALGORITMO DE TRATAMIENTO DE LAS INESTABILIDADES E-L DE ORIGEN TRAUMÁTICO}

Decimos que una muñeca presenta una inestabilidad cinética E-L cuando presenta una rotura parcial o completa de los ligamentos estabilizadores de dicha articulación que le impide sostener cargas fisiológicas sin ceder o perder su normal alineación. Si aparte de esta imposibilidad en la transmisión de cargas (inestabilidad cinética), la muñeca presenta resaltes articulares en forma de subluxaciones bruscas del escafoides, decimos que la muñeca es también cinemáticamente inestable.

En el proceso analítico que llevará a decidir un tratamiento para cada uno de estos casos, aparte de la edad del paciente, estado global de salud, y de las demandas profesionales y/o vocacionales, es preciso evaluar los siguientes cinco parámetros.

Integridad del ligamento E-L dorsal: Las lesiones parciales de los ligamentos E-L suelen afectar tan sólo al ligamento E-L anterior, con desinserción del ligamento radioescafolunar de Kuenz, y a la membrana fibrocartilaginosa E-L proximal. El ligamento E-L dorsal es mucho más resistente a la tracción que el ligamento palmar con lo que no es raro encontrarlo íntegro tras traumatismo de muñeca de baja energía. Es importante establecer hasta qué punto esta estructura esta indemne o no. Ocasionalmente el ligamento puede parecer intacto y sin embargo estar distendido de manera que permita una notable separación E-L. En otras ocasiones, el ligamento dorsal aparece avulsionado, si bien su estructura interna está conservada, permitiendo su reinserción quirúrgica. Desde un punto de vista pronóstico, la presencia o ausencia de este ligamento tiene una importancia pronóstica indudable. La maniobra de Watson, también llamada del «resalte escafoideo»y que consiste en intentar subluxar el polo proximal del escafoides por encima del borde dorsoradial del radio aplicando una fuerza hacia el dorso sobre la tuberosidad palmar del escafoides mientras se inclina radialmente la muñeca, sólo puede ser positiva si el ligamento E-L dorsal y la cápsula dorso- radial están seriamente lesionados. Las artrografías no son técnicas recomendables para evaluar el estado del ligamento E-L dorsal pues existen frecuentes perforaciones degenerativas de la membrana proximal, y sobre todo en personas de edad avanzada, que inducen a errores diagnósticos. En caso de duda, puede optarse por la artro-tomografia axial computarizada, la resonancia nuclear magnética, o mucho mejor, la artroscopia a fin de aclarar si el ligamento E-L dorsal es o no competente.

Potencial cicatricial de los ligamentos lesionados: Si los ligamentos han sufrido una lesión de tipo irregular, con un deshilachamiento importante, o si los extremos del ligamento roto están retraídos o necróticos, su potencial de cicatrización será muy pobre ${ }^{8,27}$. Por el contrario, si la lesión es del tipo avulsivo, con uno de sus extremos desinsertado del hueso correspondiente, una correcta reinserción puede lograr una cicatrización adecuada, incluso en el caso que haya pasado un cierto tiempo desde el accidente. En realidad, es más importante el parámetro «potencial cicatricial» que el hecho de que la lesión sea aguda, subaguda o crónica $^{27}$.

Integridad de los ligamentos estabilizadores secundarios: Cuando el escafoides ha perdido sus conexiones con el semilunar, puede seguir manteniendo una alineación relativamente normal gracias a la presencia de los ligamentos palmares que lo unen a la hilera distal. Es particularmente importante la estabilización que proporcionan los ligamentos escafoides-hueso grande y escafoides-trapecio-trapezoide, así como de la cápsula palmar escafo-trapecial ${ }^{4}$. Ante una disociación E-L es importante evaluar si esta «segunda línea de defensa» está o no alterada. Si lo está, el escafoides típicamente aparece colapsado en flexión-pronación, con lo que el ángulo radioescafoideo se encuentra anormalmente aumentado. El término «subluxación rotatoria del escafoides ${ }^{23}$, que a menudo se utiliza erróneamente como sinónimo de disociación E-L, sólo debe usarse para describir esta peculiar malposición del escafoides, resultado de la rotura completa de los ligamentos E-L asociada a la distensión de los estabilizadores secundarios. 
Tabla 1 - ESTAdíos CLíNICOS DE LA INESTABILIDAD E-L A PARTIR DEL ANÁLISIS DE UNA SERIE DE FACTORES QUE INFLUYEN EN EL PRONÓSTICO (VER TEXTO)

\begin{tabular}{|lcccccc|}
\hline & \multicolumn{5}{c|}{ Estadíos clínicos } \\
\cline { 2 - 7 } Factores & $\mathbf{1}$ & $\mathbf{2}$ & $\mathbf{3}$ & $\mathbf{4}$ & $\mathbf{5}$ & $\mathbf{6}$ \\
\hline El ligamento dorsal E-L es normal & Sí & No & No & No & No & No \\
\hline El ligamento dorsal E-L puede ser reparado & Sí & Sí & No & No & No & No \\
\hline La alineación carpiana es normal & Sí & Sí & Sí & No & No & No \\
\hline Hay subluxación, pero es fácilmente reducible & Sí & Sí & Sí & Sí & No & No \\
\hline No hay lesión del cartílago articular & Sí & Sí & Sí & Sí & Sí & No \\
\hline
\end{tabular}

E-L: Escafolunar

Reducibilidad de la subluxación carpiana: Cuando una subluxación E-L se ha mantenido de forma crónica, puede sufrir un proceso de fijación de la deformidad, ya sea por proliferación fibrosa o por calcificación de los extremos ligamentosos, que la convierten en irreducible. Estos focos de fibrosis típicamente aparecen entre la cara posterior-distal del escafoides y el trapezoide, y en la porción anterior de la articulación E-L diastasada ${ }^{11}$. En ocasiones la calcificación del extremo ligamentario E-L aparece cual si fuera un osteofito artrósico, que no debe confundirse con una proliferación degenerativa. Es muy importante determinar si una subluxación es reducible o no, y en qué grado, por cuanto que nunca podrá una plastia reconstructiva estabilizar una deformidad no reducible si sólo se utilizan partes blandas (capsulodesis o tenodesis). Si la reducibilidad es pobre, como se verá más adelante, es preferible optar por soluciones de tipo óseo (osteotomías o artrodesis parciales).

Estado cartilaginoso. Si existe un defecto en el cartílago articular, condrolisis reactiva, o degeneración artrósica asociada a la inestabilidad E-L (muñeca SLAC) no debe utilizarse el mismo tratamiento que si los cartílagos articulares están sanos. Las lesiones condrales pueden haber aparecido como resultado del traumatismo por efecto cizallante, o secundariamente y progresivamente como consecuencia de la anormal cinemática carpiana que se genera en estos $\operatorname{casos}^{4}$. Particularmente sensibles a desarrollar artrosis son las facetas articulares de los segmentos radio-escafoideo (zona posterior), y grande-semilunar ${ }^{3}$.

Una vez evaluados estos cinco parámetros, hay que responder en términos de «si» o «no» a las siguientes preguntas:

1) ¿Está intacto el ligamento dorsal E-L?

2) en caso de lesión E-L dorsal, ¿tiene un potencial cicatricial suficientemente bueno que permita su reparación o reinserción?

3) ¿Es normal la posición del escafoides y semilunar respecto al radio? $\mathrm{O}$ dicho de otro modo, en la radiografía de perfil ¿Están los ángulos radioescafoideo y radiolunar dentro de los límites normales, en comparación al lado contralateral?

4) en caso de que exista subluxación E-L ¿Es fácilmente reducible?

5) ¿Es normal el cartílago de las articulaciones adyacentes al escafoides y semilunar?

Si respondemos estas preguntas con un «sí» o un «no», podremos clasificar cada caso clínico dentro de alguno de los siguientes seis grupos o grados evolutivos del proceso inestabilizante E-L (Tabla 1) El hecho que el número de respuestas negativas aumente de izquierda a derecha denota la creciente severidad o cronicidad del proceso, de leve (grado 1) a severo (grado 6). 
En teoría, cada estadío merece un tratamiento proporcional a la gravedad del proceso, siguiendo el patrón siguiente:

Disociación E-L, estadío 1 (Lesión E-L incompleta): Cuando existe una rotura parcial de los ligamentos E-L ésta suele afectar al ligamento anterior y la membrana proximal. El ligamento dorsal muy raramente se ve involucrado, por lo que la alineación carpiana es normal. $\mathrm{Si}$ existe dolor, es debido a la sinovitis que resulta tras un aumento de la movilidad E-L o al efecto que pueda tener, cual cuerpo extraño, algún resto ligamentoso inestable. Es muy frecuente encontrar esta situación en el curso de una artroscopia diagnóstica. La radiografía de frente no muestra diastasis E-L, ni en posiciones extremas ni bajo carga axial, motivo por el cual algunos autores califican estos casos de «inestabilidad pre-dinámica» E-L ${ }^{8}$.

Cuando la rotura parcial se ha diagnosticado en la fase aguda, una fijación percútanea con agujas de Kirschner, idealmente bajo control artroscópico, suele ser suficiente para generar un cierto grado de artrofibrosis que resuelva la inestabilidad. Si a ello se añade una adecuada reeducación del músculo flexor carpi radialis, que es el único músculo con capacidad para controlar dinámicamente el grado de flexión del escafoides, podemos devolver al paciente un grado óptimo de funcionalidad.

Por el contrario, si la lesión se descubre en fase crónica si bien conservando íntegro el ligamento dorsal (y por tanto la normal alineación carpiana) es aconsejable desbridar artroscópicamente los extremos inestables del ligamento lesionado, avivando el borde libre articular para favorecer la subsiguiente artrofibrosis, más fijación percútanea con agujas Kirschner. La opción de añadir a todo ello un retensado capsular radioescafoideo, según Blatt, sigue siendo considerada por muchos como el tratamiento de elección de esta patología ${ }^{10,} 12$, 13, 16-18, 20. En nuestra experiencia, este tipo de capsulodesis no debe realizarse en estadíos avanzados de la inestabilidad, y salvo en raras situaciones, no debe utilizarse como único gesto terapéutico.

Disociación E-L, estadío 2 (Lesión E-L completa, reparable, sin desalineación car- piana): Este estadío se caracteriza por la presencia de una lesión completa de todos ligamentos E-L, si bien el ligamento dorsal, el más importante desde un punto de vista biomecánico, puede todavía ser reparado o reinsertado con buenas opciones de éxito, es decir con un buen potencial cicatrizante. Por definición, en este tipo de lesión puede no existir ni colapso carpiano, ni desaxación alguna gracias a la presencia de unos buenos ligamentos estabilizadores secundarios (principalmente el escafoides-hueso grande y el escafoides-trapecio-trapezoide). En cambio, sí puede aparecer una diastasis entre el escafoides y el semilunar bajo carga axial, en la proyección con el puño apretado, o en determinadas posiciones de la muñeca.

Si el ligamento puede reinsertarse o repararse con éxito, éste es el camino a seguir. Ello, por supuesto, convierte el estadío 2 en un estadío 1, implicando la necesidad, ya mencionada para ese estadío, de una fijación percútanea más reeducación del flexor carpi radialis.

Un típico ejemplo de este estadío es la lesión por hiperextensión de la muñeca con rotura completa de los ligamentos escafolunares, $y$ que sin embargo aparecen sin diastasis E-L. Si la lesión ligamentosa es del tipo avulsivo, puede lograrse un excelente resultado con la reinserción con puntos transóseos o anclajes metálicos de los ligamentos avulsionados ${ }^{18}$.

Disociación E-L, estadío 3 (Lesión E-L completa, no reparable, sin desalineación carpiana): El tercer estadío se caracteriza por la presencia de una rotura completa de todos los ligamentos E-L, siendo el ligamento dorsal no reparable o con poca capacidad cicatricial. Si las estructuras estabilizadoras secundarias (ligamentos escafoides-hueso grande, escafoidestrapecio-trapezoide, escafoides-piramidal dorsal, así como la cápsula palmar escafo-trapecial) se mantienen intactas, el escafoides puede mantener una normal alineación con respecto al radio y el hueso grande. Puede no existir diastasis E-L y que solo aparezca alguna anomalía en la alineación carpiana en determinadas posiciones y/o condiciones de carga (inestabilidad dinámica). En tales circunstancias puede estar indicada la utilización de injertos hueso-ligamento-hueso, si bien es importante saber que 
sus resultados a largo plazo son todavía desconocidos $^{21,22}$.

Disociación E-L, estadío 4 (Lesión E-L completa, no reparable, con subluxación carpiana fácilmente reducible): Este estadío se caracteriza por una pérdida completa de la relación E-L, incluyendo arrancamiento del ligamento escafo-piramidal dorsal, más elongación de los ligamentos que conectan el escafoides con la hilera distal del carpo. El escafoides presenta la típica subluxación rotatoria en flexionpronación (ángulo radioescafoideo $>45^{\circ}$ ) mientras que el semilunar aparece anormalmente extendido (DISI) y parcialmente desplazado hacia el cúbito. Para que esto último suceda es preciso que exista una cierta elongación de los ligamentos radio-semilunares (corto y largo). A todo ello hay que añadir una característica esencial: es preciso que la subluxación E-L sea fácilmente reducible y que no existan lesiones traumáticas o degenerativas en el cartílago articular. En estos casos está indicada la técnica «3LT» que se describirá a continuación.

Disociación E-L, estadío 5 (Lesión E-L completa, no reparable, con subluxación carpiana irreducible, y cartílago normal): Cuando una subluxación E-L se ha mantenido por un tiempo prolongado, la cápsula articular puede presentar fenómenos de retracción, con calcificación de los extremos ligamentosos lesionados, más fibrosis de los espacios que han quedado vacíos como consecuencia del colapso carpiano. Todo ello induce a un estado de irreductibilidad, que impide que las técnicas de reconstrucción antes mencionadas puedan utilizarse con éxito. Sin embargo, si los cartílagos articulares se mantienen intactos, pueden plantearse técnicas estabilizadoras menos radicales que las que deben utilizarse cuando ya existe artrosis post-traumática. Entre las técnicas más comúnmente utilizadas en el estadío 5 destacan las artrodesis parciales escafoides-trapecio-trapezoide, o escafoides-hueso grande $\mathrm{e}^{23-25}$.

Disociación E-L, estadío 6 (Lesión E-L completa, no reparable, con subluxación carpiana irreducible, $y$ arthrosis post-traumática): Una disfunción crónica E-L conlleva a medio o largo plazo la aparición de cambios de- generativos empezando casi siempre a nivel de la apófisis estiloides radial, progresando hasta involucrar todo el espacio radioescafoideo y siguiendo hacia la articulación semilunar-hueso grande. Es la artrosis denominada SLAC ( $«$ Scaphoid-Lunate Advanced Collapse»), con un patrón de progresión degenerativa muy característico, cuyo tratamiento debe establecerse en función del grado y la extensión de la degeneración existente. ${ }^{3}$ El objetivo del tratamiento en tales casos es eliminar el dolor a expensas de una pérdida de movilidad inevitable, ya sea mediante la exéresis de toda la hilera proximal del carpo o por la resección del escafoides más artrodesis mediocarpiana ${ }^{24,26 .}$

Reconstrucción de los ligamentos escafolunares mediante injertos tendinosos. Revisión de la Literatura.

En 1978 Palmer y colaboradores ${ }^{15}$ publicaron por primera vez algunos resultados obtenidos tras la reconstrucción de los ligamentos EL mediante fragmentos de tendón. La técnica inicial, descrita por Dobyns, consistía en la creación de túneles óseos anteroposteriores a través del polo proximal del escafoides y del semilunar, por los cuales se pasaba un fragmento de tendón extensor carpi radialis longus (ECRL), en forma de bucle, que se suturaba fuertemente sobre sí mismo dando una muy rígida estabilización primaria. Desafortunadamente, la zona por donde transcurrían dichos túneles está muy pobremente vascularizada con lo que no era infrecuente ver fracturas a ese nivel, con necrosis y degeneración progresiva de los cartílagos adyacentes, por lo que su práctica fue más tarde desaconsejada ${ }^{15}$.

Unos años más tarde, Almquist y colaboradores ${ }^{9}$ presentaron mejores resultados a corto plazo con la técnica denominada de los «cuatro huesos». Según dicha técnica, un fragmento de tendón del músculo extensor carpi radialis brevis cruzaba el hueso grande del dorso a la palma para penetrar en el semilunar de delante a atrás, y pasando después por el escafoides de atrás adelante. Con ello se reconstruía el ligamento E-L dorsal. Una vez de nuevo en la palma el tendón se utilizaba para reforzar los ligamentos radiocarpianos anteriores. No existía un bucle tendinoso E-L, sino una recons- 
trucción más compleja que cruzaba verticalmente los espacios mediocarpiano y radiocarpiano anterior. El principal problema de esa técnica fue precisamente debido a la excesiva fibrosis que se creaba, con la correspondiente limitación global de la movilidad de la muñeca, aparte de los problemas relativos al hecho de practicar túneles en zonas tan pobremente vascularizadas. Como pasó con otras técnicas parecidas, nunca aparecieron publicaciones analizando sus resultados a largo plazo.

En 1992 Linscheid y Dobyns ${ }^{14}$ propusieron una nueva técnica estabilizadora del escafoides mediante una bandeleta de tendon ECRL que se mantenía insertada distalmente en la base del segundo metacarpiano para atravesar el escafoides de atrás adelante, para emerger en la tuberosidad anterior del mismo. Una vez en la palma, el tendón pasaba al interior de la cavidad radioescafoidea, y a nivel de la apófisis estiloides radial rodeaba al cuello del escafoides siguiendo su cresta dorsoradial, hasta reconstruir el ligamento E-L dorsal. Nunca se publicaron resultados ni a corto plazo de una técnica que, en teoría, cumplía todos los requisitos para una óptima estabilización tanto del polo distal-anterior del escafoides, como del dorso de la articulación E-L.

En 1995 Brunelli y Brunelli ${ }^{11}$ propusieron una técnica parecida a la de Linscheid y Dobyns, si bien en este caso se utilizaba una bandeleta del flexor carpi radialis (FCR) que, atravesando de la palma al dorso al escafoides por su tercio distal se impedía su subluxación en flexión. El tendón, una vez en la cara posterior, se anclaba al dorso del radio, reforzando así la cápsula radioescafoidea dorsal, a modo de capsulodesis tipo Blatt ${ }^{10}$, estabilizando aún más si cabe al escafoides. Dicho método fue subsiguientemente modificado por Van den Abbeele y colaboradores ${ }^{19}$ quienes sugirieron que el tendón no cruzara la articulación radiocarpiana sino que podía reinsertarse sobre el dorso del semilunar con anclajes metálicos.

Existen diferencias substanciales entre las tenodesis más recientemente recomendadas y los métodos sugeridos en los años 70 . En las técnicas más recientes ${ }^{11,19}$ no sólo se intenta estabilizar el polo proximal del escafoides en relación al semilunar, sino que se enfatiza la nece- sidad de reforzar la cápsula distal-palmar escafoides-trapecio. Es más, los túneles óseos no cruzan el polo proximal del escafoides, cuya vascularización es pobre, sino que lo atraviesan por su polo distal, con lo que se evitan la necrosis y los colapsos óseos tan frecuentes con las técnicas antiguas.

La técnica que se describe a continuación también utiliza una bandeleta del FCR. En este caso, no obstante, se busca actuar sobre el carpo en cuatro sentidos: 1) para reforzar las conexiones del escafoides con la hilera distal del carpo, 2) para reconstruir de una manera bastante anatómica el ligamento E-L dorsal, 3) para mejorar las posibilidades de cicatrización del tendón en el semilunar, y 4) para reducir y estabilizar la relación entre el radio y el bloque semilunar-piramidal en una posición fisiológicamente correcta. Dado que la tenodesis que proponemos refuerza o substituye a tres ligamentos (escafoides-trapecio-trapezoide, E-L dorsal y radiopiramidal dorsal) la hemos denominado técnica «3LT» («three-ligament-tenodesis») y está particularmente indicada en las disociaciones E-L estadío 4, según el esquema descrito más arriba, es decir en subluxaciones carpianas por rotura completa de los ligamentos E-L, no reparables, reductibles y con un cartílago articular intacto. La técnica no es nueva, sino que incorpora aspectos de las técnicas antes descritas por Linscheid y Dobyns ${ }^{14}$, Brunelli y Brunelli ${ }^{11}$, y Van den Abbeele y colaboradores $^{19}$, aparte de introducir ciertos detalles nuevos que, en nuestra experiencia, aseguran su mejor resultado a medio plazo.

\section{TÉCNICA QUIRÚRGICA}

Se practica una incisión de $8 \mathrm{~cm}$ en el dorso de la muñeca, ya sea en zig-zag o longitudinal, atravesando la piel y el tejido subcutáneo sobre el tubérculo de Lister. Se identifican y protegen las ramas sensitivas dorsales del nervio radial y cubital si aparecen en la vecindad de la incisión. Se secciona el retináculo extensor (ligamento anular dorsal) siguiendo el tercer compartimento que contiene el tendón extensor pollicis longus. Los septos que separan los compartimentos segundo, cuarto y quinto son sec- 
cionados frontalmente de manera que puedan protegerse todos los tendones extensores que cruzan la muñeca. Los dos colgajos de tejido retinacular así creados se reclinan hacia radial y cubital. La mayoría de estos septos contienen vasos septales que deben coagularse para evitar un sangrado excesivo en el postoperatorio.

La cápsula dorsal, ahora expuesta, contiene en su interior el ligamento radiopiramidal dorsal, de disposición oblicua, casi paralela a las fibras del retináculo extensor, uniendo el borde dorsomedial del radio con la cresta dorsal del piramidal. La incisión capsular debe iniciarse en el piramidal y seguir la dirección de este ligamento, separando sus fibras pero manteniendo una buena porción del mismo unida al radio y al piramidal. Cuando llega al radio, la incisión cambia de dirección continuando por su borde dorsal hasta la estiloides radial, desinsertando así completamente la cápsula radioescafoidea . A continuación se realiza otra incisión capsular paralela a esta última, empezando en el borde proximal del trapecio, siguiendo las fibras horizontales del ligamento transversal dorsal del carpo, a nivel del cuello del hueso grande, para converger con la anterior a nivel del dorso del piramidal. Las dos incisiones deben encontrarse creando un colgajo capsular de base radial que se levantará separando cuidadosamente sus conexiones con los huesos de la hilera proximal, según la técnica descrita por Berger,Bishop y Bettinger ${ }^{28}$. Dado que la incisión proximal sobre el borde dorsal del radio secciona necesariamente el nervio interóseo posterior, es preferible extirpar dicho nervio completamente dejando su muñón a nivel de la membrana interósea radiocubital donde un eventual neuroma estaría mejor protegido que en la vecindad de la articulación radiocarpiana.

Una vez expuesta la cara dorsal de los huesos escafoides, semilunar y hueso grande, se inspeccionan los diferentes espacios articulares a fin de decidir si está indicada la técnica que se describe a continuación. Para ello se analizan los factores antes citados: extensión del daño ligamentoso-capsular, reducibilidad, y estado del cartílago articular. La reducibilidad se explora mediante tracción axial, o por manipulación directa, o con la ayuda de agujas Kirschner que actúan a modo de pequeñas palancas que facilitan la movilización de los huesos. En este último caso, si para cerrar el espacio E-L se necesita tanta fuerza como para doblar una aguja de Kirschner (K) de 1,5 mm de diámetro, decimos que el caso no es reducible. Sólo seguiremos adelante con la técnica 3LT si la subluxación es fácilmente reducible y no existe grado alguno de lesión cartilaginosa en la zona de carga del escafoides y/o del hueso grande.

Se inserta una aguja-guía en el escafoides, penetrando por su borde dorsal-interno donde originariamente se encuentra el ligamento E-L dorsal. Dicha aguja-guía se dirige, siguiendo el eje principal del escafoides, hacia el vértice anterior de su tuberosidad distal. Al tener una visión directa de los espacios articulares escafoides-hueso grande y radio-escafoides, es muy improbable que se penetre con la aguja dichas articulaciones. De todos modos, siempre es recomendable realizar un control radioscópico que asegure la correcta situación de la aguja, antes de labrar el túnel transescafoideo definitivo con una broca canulada de $3.2 \mathrm{~mm}$ de diámetro.

Con la punta de la fresa emergiendo por la cara palmar como referencia, practicamos una incisión palmar transversal de $1.5 \mathrm{~cm}$ de longitud. A través de esta incisión se identifica el punto de salida de la broca, y se localiza la vaina del tendón FCR que debe estar en la vecindad del túnel que hemos realizado. Se obtiene a continuación una bandeleta de unos $8 \mathrm{~cm}$ de longitud del tendón FCR ya sea mediante una única incisión longitudinal, o más comúnmente mediante pequeñas incisiones transversales sobre su trayecto. La bandeleta debe tener un diámetro no superior a los $3 \mathrm{~mm}$, a fin de facilitar su paso por el túnel del escafoides, ni ser excesivamente delgada pues difícilmente seria incorporado en el callo óseo intraescafoideo si existiera movilidad entre el tendón y el hueso. La vaina del tendón FCR, que a ese nivel se hace profundo siguiendo por detrás la cresta medial del trapecio, no se abre. Con ello se asegura que exista a ese nivel un cambio de orientación del tendón, de dentro a fuera, que remeda el trayecto del ligamento escafoides-hueso grande. El tendón no debe pasarse superficial a la rama tenar de la arteria radial, que en este punto cruza la incisión, sino que debe rodear la 

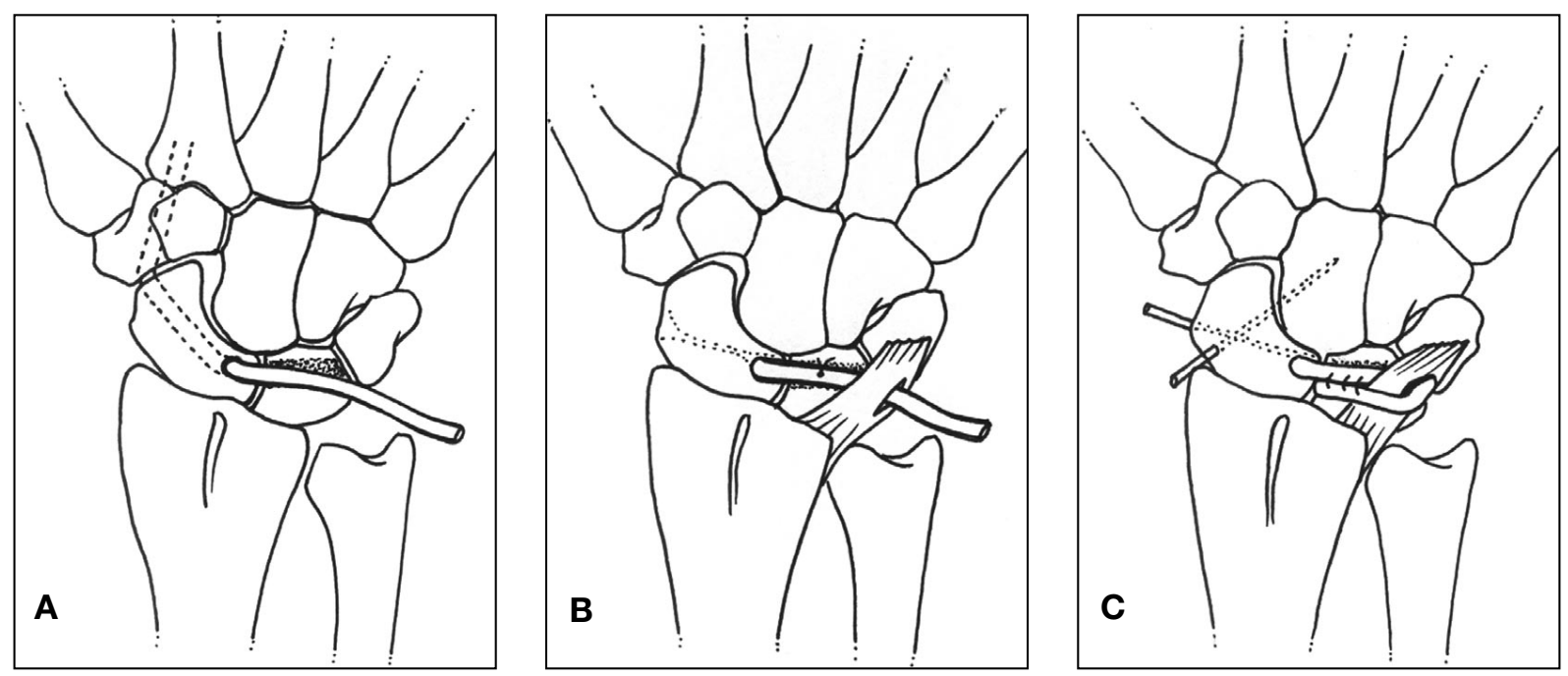

Figura 1: Representación esquemática de la técnica 3 LT vista desde el dorso. A: La bandeleta del tendón FCR atraviesa al escafoides, de distal a proximal, y de palmar a dorsal, siguiendo el eje longitudinal del mismo. Se decortica el dorso del semilunar en forma de trinchera a fin de facilitar la incorporación del tendón al hueso. B: La bandeleta tendinosa utiliza al ligamento radiopiramidal dorsal como punto de apoyo para su tensado. C: El extremo tendinoso, una vez tensado, se sutura sobre si mismo, no sin antes bloquear con agujas $\mathrm{K}$ las articulaciones escafoides-hueso grande y escafoides-semilunar.

tuberosidad del escafoides, adyacente al mismo, hasta penetrar en el túnel antes creado. El paso de la bandeleta tendinosa por el escafoides se realiza mediante la utilización de una pinza pasa-tendón, o bien mediante un pequeño bucle alámbrico introducido desde el dorso del escafoides.

Cuando la bandeleta del tendón está ya en el dorso, se procede a preparar la zona de inserción del mismo en el semilunar, decorticando una franja transversal de unos $3 \mathrm{~mm}$ de ancho sobre su borde dorsal, mediante el uso de una pinza-gubia. Esa especie de trinchera debe ser suficientemente profunda como para descubrir hueso esponjoso. Mediante la introducción de un anclaje metálico (Mitek® Mini QuickAnchor del 1.8, o similar) se asegura que el tendón estará adosado permanentemente al hueso esponjoso, garantizándose así una sólida unión hueso-tendón una vez que éste se coloque en dicha trinchera.

A continuación se localiza el extremo distal e interno del ligamento radiopiramidal dorsal. Manteniendo su extremo distal sólidamente unido al piramidal, con una pinza tipo mosquito se crea un túnel adyacente al polo proximal del piramidal, por un plano profundo al ligamento an- tedicho, emergiendo a nivel del trayecto del tendón extensor carpi ulnaris. La bandeleta se hace pasar por ese túnel lo cual permitirá su retensado utilizando el ligamento radiopiramidal dorsal como polea.

Antes de colocar ninguna sutura, se procede a la reducción de la subluxación de escafoides respecto al radio (ángulo radioescafoideo $<45^{\circ}$ ), y a la del semilunar respecto escafoides (ángulo escafolunar $\approx 60^{\circ}$ ). Como queda dicho más arriba, la reducción resulta más fácil si previamente se han introducido agujas $\mathrm{K}$ en el dorso del escafoides y del semilunar que permitan su fácil realineación. Una vez obtenida, la reducción se asegura con otras dos agujas $\mathrm{K}$ percutáneas: una, oblicua, de dorsal a palmar y de fuera a dentro, entre el escafoides y el hueso grande; y otra, transversal, de palmar a dorsal y de fuera a dentro, entre el escafoides y el semilunar. En este punto se recomienda comprobar la calidad de la reducción y la situación de las agujas mediante radioscopia.

A continuación se tira del extremo libre de la bandeleta tendinosa, utilizando el ligamento radiopiramidal dorsal como polea. El tendón debe quedar hundido en la trinchera del semilunar, posición que aseguraremos mediante la su- 

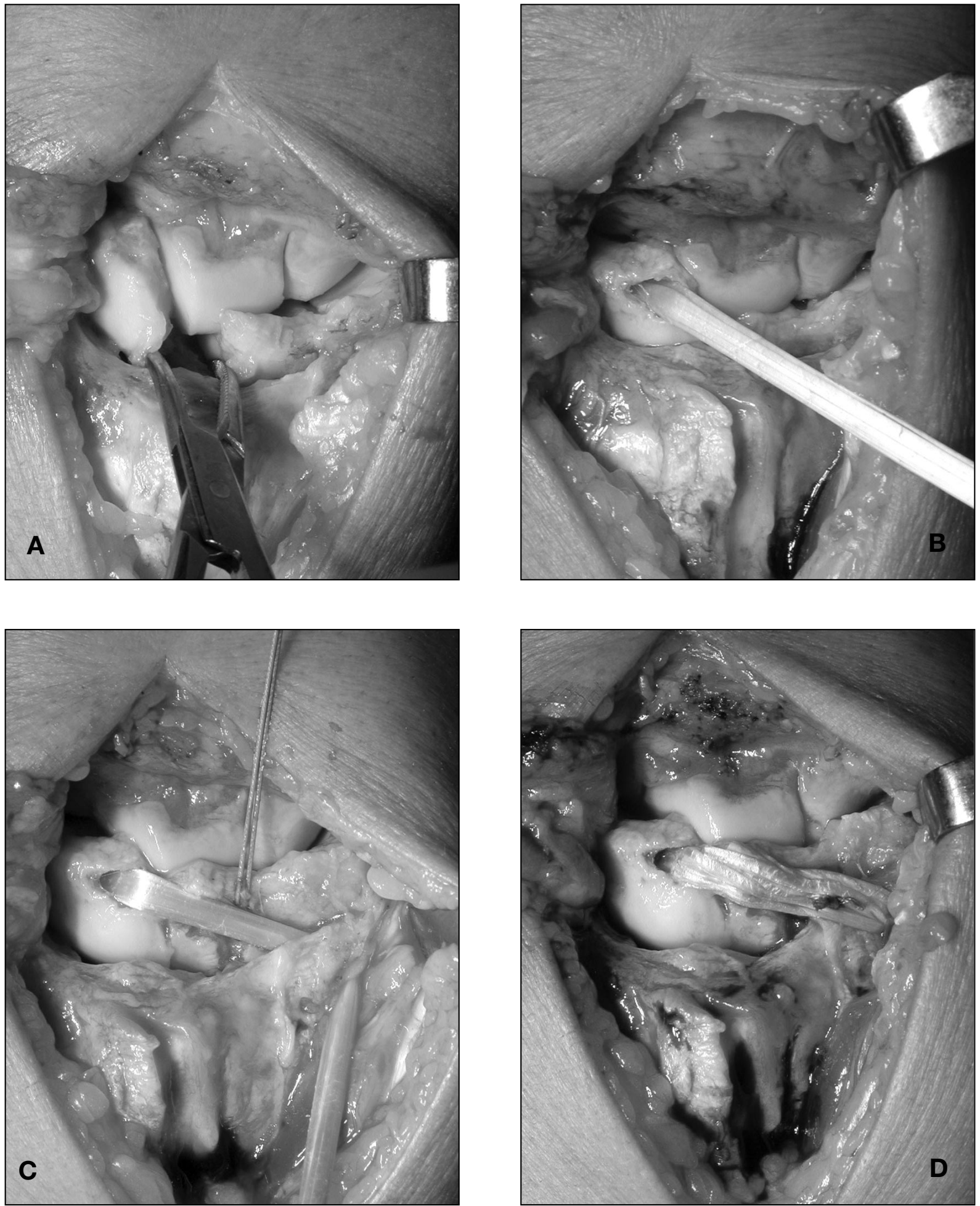

Figura 2: Fotografías quirúrgicas de un caso de inestabilidad escafolunar de más de 8 meses de evolución tratado mediante la técnica 3LT. A: Se comprueba la rotura completa de todos los ligamentos escafolunares, así como el pobre potencial cicatrizante que poseen los ligamentos lesionados. B: La bandeleta tendinosa emergiendo por el borde dorsocubital del escafoides. $C$ : El tendón pasa por un plano profundo rodeando al ligamento radiopiramidal dorsal. En su trayecto por el dorso del semilunar, el tendón queda enterrado en la trinchera previamente labrada. En esta posición se mantendrá gracias a la sutura unida al anclaje metálico que se le aplicará a continuación. D: Aspecto final de la reconstrucción escafolunar, antes del cierre del colgajo capsular. 
tura del mismo al anclaje metálico previamente colocado. Sin soltar la tracción, el extremo del tendón se sutura sobre si mismo con material no reabsorbible de $3 / 0$, con lo cual la tensión del injerto no depende del anclaje metálico solamente sino que se traslada al punto de inflexión del tendón sobre el ligamento radiopiramidal dorsal. Con ello se asegura una correcta reducción del bloque semilunar-piramidal en relación al radio.

Una vez comprobada la idoneidad de la reconstrucción ligamentosa, se cierra el colgajo capsular mediante suturas reabsorbibles uniendo las fibras adyacentes de los ligamentos radiopiramidal y transversal dorsal del carpo anteriormente separadas. Se colocan además algunos puntos de sutura uniendo el colgajo con el ligamento reconstruido para asegurar su incorporación a la cápsula, así como puntos de anclaje del colgajo al borde dorsal de la apófisis estiloides radial. A continuación de repara el retináculo extensor, se colocan drenajes y se cierra el plano cutáneo.

La muñeca se inmoviliza mediante dos férulas de yeso almohadillado en posición neutra, sin incluir ni el pulgar ni el codo. Dicho vendaje se cambia a los 10 días por un yeso antebraquial hasta alcanzar un total de 6 semanas de inmovilización, momento en que se coloca una muñequera con férula palmar que se retirará tres veces al día para realizar movimientos activos de circundución radiocarpiana. A las 8 semanas se retiran las agujas y, todavía con la protección de la muñequera entre sesiones, puede iniciarse una rehabilitación funcional más intensa. A los 3 meses puede prescindirse de la muñequera, si bien no se permitirán deportes de contacto (fútbol, básquetbol, esquí...) hasta los 6 meses de la intervención. Es importante hacer notar a los fisioterapeutas que se encarguen de estos casos que, aunque típicamente esta intervención genera una importante fibrosis capsular, y por tanto una limitación al principio importante de la flexión palmar de la muñeca, deben evitarse las movilizaciones forzadas pasivas en flexión. Efectivamente, la fibrosis puede tardar más de un año en lograr su normal elasticidad, sin que ello pueda acelerarse (sino todo lo contrario) mediante manipulaciones intempestivas bajo anestesia.

\section{RESULTADOS}

Hemos revisado recientemente 38 pacientes ( 24 hombres; 14 mujeres, edad promedio 31 años, entre 18 y 52) cuya inestabilidad E-L fue tratada mediante el método 3LT. Veintiún pacientes presentaban una disociación E-L en estadío 3, ocho en estadío 4, y nueve en estadío 5. A estos últimos se les aplicó la técnica 3LT tras comprobar que la subluxación, inicialmente irreducible, se convertía en reducible tras extirpar la fibrosis existente entre el escafoides y el trapezoide, y entre el escafoides y el semilunar.

Con un seguimiento promedio de 46 meses (rango 7-98), todos los pacientes fueron interrogados acerca del grado de dolor residual y limitación funcional. Se determinó su movilidad, fuerza de garra y pinza. Además, se obtuvieron radiografías de frente y perfil de la muñeca intervenida a fin de comprobar si existía diastasis E-L residual, desalineación carpiana y/o artrosis.

Veintiocho pacientes declararon no tener ningún tipo de molestias en reposo, si bien sólo tres declaraban no tenerlas ni al realizar esfuerzos considerables con la mano afectada. Ocho pacientes presentaban ligeras molestias en reposo y dolor a medianos esfuerzos, mientras que en dos ocasiones existían molestias continuas con brotes de inflamación tras mínimos esfuerzos.

Veintinueve pacientes pudieron reincorporarse a su trabajo original sin limitaciones. En siete ocasiones se registró una cierta reducción de su capacidad funcional si bien ello no les impidió volver a sus ocupaciones habituales, mientras que dos tuvieron que aceptar un grado de incapacidad incompatible con su trabajo.

Los rangos promedio de movilidad pasiva residual fueron de $51^{\circ}$ de flexión (74\% respecto a la movilidad del lado opuesto), $52^{\circ}$ de extensión (77\%), 15을 de inclinación radial (78\%) y $28^{\circ}$ de inclinación cubital $(92 \%)$. La fuerza de garra medida con un dinamómetro Jamar en posición 3, fue del $65 \%$ respecto al lado contralateral.

No se apreció ningún caso de necrosis del escafoides. En la mayoría existió un ligero aumento de la diastasis E-L (3.8 $\mathrm{mm}$ de promedio) en relación del observado tras la interven- 

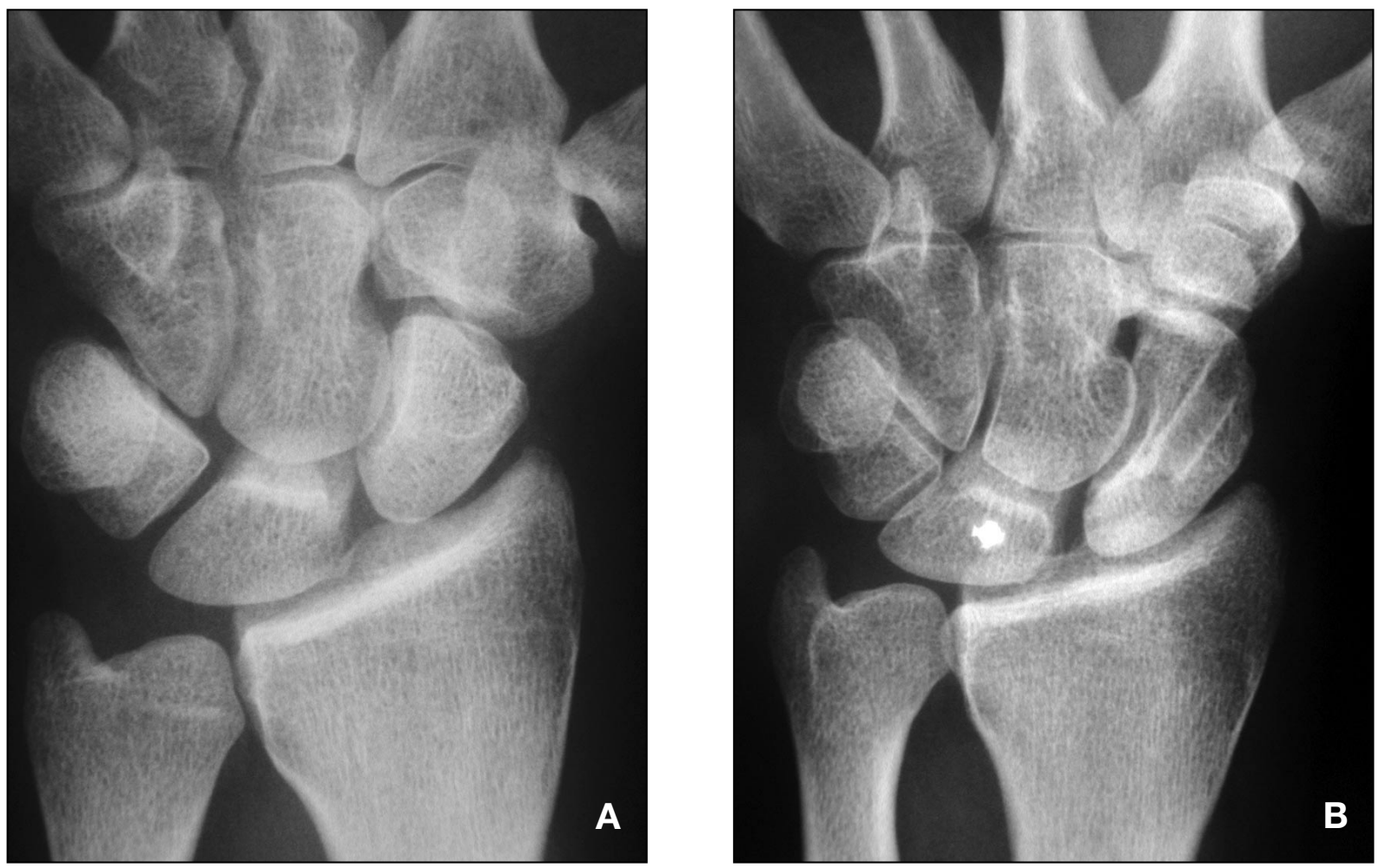

Figura 3: Imagen radiográfica antes de la intervención (A), y a los 3 años, donde se comprueba una correcta verticalidad del escafoides y una diastasis escafolunar aceptable (B). El paciente presenta una función muy buena, sin molestias, con una pérdida de flexión inferior al 20\%, y una fuerza de garra del $85 \%$.

ción $(3.1 \mathrm{~mm})$, siendo sin embargo inferior al existente antes de la intervención, donde la mayoría presentaban más de $5 \mathrm{~mm}$ de diastasis E-L. No se observó ninguna disminución significativa de la altura carpiana, y tan sólo en dos casos se observó la presencia, ya en el post-operatorio inmediato, de una desalineación en VISI, por exceso en la reducción. Siete pacientes presentaron signos de progresión artrósica radioescafoidea, si bien ninguno de ellos declaró tener mayores molestias que los pacientes sin este hallazgo. Solo en dos casos la técnica fracasó completamente, apareciendo un patrón de artrosis avanzada, tipo SLAC 2. Ninguno de ellos precisó, todavía, ser reintervenido.

A tenor de estos resultados, y en comparación con los publicados con otras técnicas, nos permitimos aconsejar esta intervención, si bien con la reserva que impone el que todos los casos evolucionaran hacia una situación muy aceptable, si bien casi nunca normal. 


\section{BIBLIOGRAFÍA}

1. Walsh, J.J.; Berger, R.A.; Cooney, W. P.: Current status of scapholunate interosseous ligament injuries. J. Am Acad Orthop Surg 2002; 10: 32-42.

2. Mink van der Molen, A. B., Groothoff, J. W.; Visser, G. J. P. et al.: Time off work due to scaphoid fractures and other carpal injuries in the Netherlands in the period 1990 to 1993. J. Hand Surg 1999; 24B: 193198.

3. Watson, H. K.; Ballet, F. L.: The SLAC wrist: Scapholunate advanced collapse pattern of degenerative arthritis. J. Hand Surg 1984; 9A: 358-365.

4. Short, W. H.; Werner, F. W.; Green, J. K. et al. Biomechanical evaluation of ligamentous stabilizers of the scaphoid and lunate. J. Hand Surg 2002; 27: 991-1002.

5. Linscheid, R. L.; Dobyns, J. H.; Beabout, J. W. et al.: Traumatic instability of the wrist: Diagnosis, classification, and pathomechanics. J. Bone Joint Surg 1972; 54A: 1612-1632.

6. Taleisnik, J.: The Wrist. New York: Churchill Livingstone, New York, 1985: 229-328.

7. Stanley, J. K.; Trail, I. A.: Carpal instability. J. Bone Joint Surg 1994; 76B: 691-700.

8. García-Elías, M.; Geissler, W. B.: Carpal Instabilities. En Green D. P.; Hotchkiss, R. N.; Pederson, W. C. y Wolfe, S. W. (eds.): Green's Operative Hand Surgery. $5^{\mathrm{a}}$ ed. ElsevierChurchill Livingstone 2005: 535-604.

9. Almquist, E. E.; Bach, A. W.; Sack, J. T., et al.: Four-bone ligament reconstruction for treatment of chronic complete scapholunate separation. J. Hand Surg 1991; 16A: 322327.

10. Blatt, G.: Capsulodesis in reconstructive hand surgery.
Dorsal capsulodesis for the unstable scaphoid and volar capsulodesis following excision of the distal ulna. Hand Clin 1987; 3: 81-102.

11. Brunelli, G. A.; Brunelli, G. R.: A new technique to correct carpal instability with scaphoid rotary subluxation: A preliminary report. J Hand Surg 1995; 20A: S82-S85.

12. Busse, F.; Felderhoff, J.; Krimmer, H. et al.: Scapholunate dissociation: treatment by dorsal capsulodesis Handchir Mikrochir Plast Chir 2002; 34: 173-181.

13. Deshmukh, S. C.; Givissis, P.; Belloso, D. et al.: Blatt's capsulodesis for chronic scapholunate dissociation. J. Hand Surg 1999; 24B: 215-220.

14. Linscheid, R. L.; Dobyns, J. H.: Treatment of scapholunate dissociation. Hand Clin 1992; 8: 645-652.

15. Palmer, A. K.; Dobyns, J. H.; Linscheid, R. L.: Management of post-traumatic instability of the wrist secondary to ligament rupture. J Hand Surg 1978; 3: 507-532.

16. Slater, R. R.; Szabo, R. M.; Bay, B. K. et al.: Dorsal intercarpal ligament capsulodesis for scapholunate dissociation: Biomechanical analysis in a cadaver model. J. Hand Surg 1999; 24A: 232-239.

17. Szabo, R. M.; Slater, R. R.; Palumbo, C. F. et al.: Dorsal intercarpal ligament capsulodesis for chronic, static scapholunate dissociation: Clinical results. J. Hand Surg 2002; 27 : 978-984.

18. Uhl, R. L.; Williamson, S. C.; Bowman, M. W. et al.: Dorsal capsulodesis using anchors. Am J. Orthop 1997; 26: 547548.

19. Van Den Abbeele, K. L. S., Loh, Y. C.; Stanley, J. K.; et al.: Early results of a modified Brunelli procedure for scapholunate instability. J. Hand Surg 1998; 23B: 258-261.

20. Wintman, B. I.; Gelberman, R. H.; Katz, J. N.: Dynamic scapholunate instability: Results of operative treatment with dorsal capsulodesis. J. Hand Surg 1995; 20A: 971-979.

21. Weiss, A. P. C.: Scapholunate ligament reconstruction using a bone-retinaculum-bone autograft. J. Hand Surg 1998; 23A: 205-215.

22. Harvey, E. J.; Hanel, D. P.: Bone-ligament-bone reconstruction for scapholunate disruption. Tech Hand Upper Extr Surg 2002; 6: 2-5.

23. Watson, H. K.; Ryu, J.; Akelman, E.: Limited triscaphoid intercarpal arthrodesis for rotatory subluxation of the scaphoid. J. Bone Joint Surg 1986; 68A: 345-349.

24. Watson, H. K.; Weinzweig, J.; Guidera, P. M. et al.: One thousand intercarpal arthrodeses. J. Hand Surg 1999; 24B: 307315.

25. Young Szalay, M. D.; Peimer, C. A.: Scaphocapitate arthrodesis. Tech Hand Upper Extrem Surg 2002; 6: 56-60.

26. Tomaino, M. M.; Miller, R. J.; Cole, I.; Burton, R. I.: Scapholunate advanced collapse wrist: Proximal row carpectomy or limited wrist arthrodesis with scaphoid excision? J. Hand Surg 1994; 19A: 134142.

27. Larsen, C. F.; Amadio, P. C.; Gilula, L. A.; et al.: Analysis of carpal instability: I. Description of the écheme. J. Hand Surg 1995; 20A: 757-764.

28. Berger, R. A.; Bishop, A. T.; Bettinger, P. C.: New dorsal capsulotomy for the surgical exposure of the wrist. Ann Plast Surg 1995; 35: 54-59. 